\title{
Tracing Public Opinion Propagation and Emotional Evolution Based on Public Emergencies in Social Networks
}

\author{
H. Wei-dong, W. Qian, C. Jie
}

\author{
Huang Wei-dong* \\ Nanjing University of Posts and Telecommunications \\ Nanjing, Jiangsu, Peoples R China \\ *Corresponding author:huangwd@njupt.edu.cn

\section{Wang Qian} \\ Nanjing University of Posts and Telecommunications \\ Nanjing, Jiangsu, Peoples R China \\ 18262621679@163.com

\section{Cao Jie} \\ Nanjing University of Information Science and Technology \\ Nanjing, Jiangsu, Peoples R China. \\ cj@amss.ac.cn
}

\begin{abstract}
Social network has become the main communication platform for public emergencies, and it has also made the public opinion influence spread more widely. How to effectively obtain public opinions from it to guide the healthy development of the society is an important issue that the government and other functional departments are concerned about. However, the interaction and evolution mechanism between the subject and the environment in the public opinion propagation is complicated, and the public and media attention and reaction to the incident are closely linked with the progress of the incident disposal. And public mining corpus has some shortcomings in the distribution of emotional classification. Only the timely update of artificial rules and emotional dictionary resources, it can handle new text data well. In fact, from the perspective of public opinion propagation, this paper built the network matrix between Internet users through the forwarding relationship, and used the social network analysis method and the emotion mining analysis technology to study the interaction and evolution mechanism between the subject and the environment in the public opinion propagation, and it studied the role of users in the emotional propagation of social networks. This paper proposed a sentiment analysis method on the micro-blog platform, which expanded the emotional dictionary and took sentence and emoticon and sentence patterns into account, which improved the accuracy of positive and negative classifications and emotional polarity analysis of the micro-blog.
\end{abstract}

Keywords: opinion mining, the public opinion propagation, semantic classes, machine learning.

\section{Introduction}

People gather on social networking sitesdue to the similar values and habits, and they become the realistic social digital projection. Based on modern information dissemination of digital social network, on the one hand, information dissemination speed and efficiency of social network have been greatly enhanced; on the other, information sharing based on social relations provides an effective filtering mechanism to spread a lot of information on the Internet. Thus, social networking sites become the most important platform for information sharing and dissemination [3]. 
Public opinion phenomenon on social network and its development laws are very rich and complicated. Especially, the subject of social network is the people who are with highly intelligence and adaptive capacity, and its cognition and decision-making behavior is the adaptive process of continuous evolutionary learning, and they changed their behavior through interaction with other subjects as well as the environment [19]. This complex adaptive and learning mechanism is projected to digital social network, and its propagation mechanism has the dynamic characteristics of the time-varying dynamic evolution, complexity and adaptability in the subject created more profound complexity of the system [18]. Therefore, it is necessary to explore an effective method to elaborate its run and propagation mechanism. And netizens are always generating massive amounts of data. With the characteristics of rapid growth, structural diversity [21], dynamic updating and wide ranges, these data contains public emotional information of all levels of society. Mining the public emotional information makes sense for the research of information retrieval, electronic commerce and public opinion supervision.

In recent years, scholars have carried out relevant research on the public opinion propagation mechanism. FeiXiong [16] studied the diffusion of micro-blog information based on forwarding mechanism and proposed the information diffusion model and verified the correctness of the model by micro-blog hot event. Lee [5] applied density-based online clustering method to mine text flow to assess the influence of public opinion events to achieve the goal of situational awareness and risk management; Zhao Haiqing [22] analyzed the nature of the complex network of public opinion propagation, the study found that: the key node in the core position has a high degree of activity and participation, which should be highly valued by network public opinion monitors and leaders; Su Xiaoping [9] put forward a local central measurement method, and the measurement results show that in the social network, the importance evaluation of nodes depends on both the centrality of the nodes in the community and "bridging" nature between the community.

At present, the researches on emotional analysis mainly focus on the emotion excavation and calculation of the information released by Internet users in the Internet [12], [13], [14], as well as the analysis the related factors of emotion, such as the stock market volatility the political election results and movie box office, which are predicted by Internet emotions. Riloff [8] proposed to construct a emotion dictionary based on a large number of corpus statistical analysis in order to classify emotion; Wiebe [15] added part-of-speech analysis based on the emotion dictionary and completed the subjective and objective classification of corpus based on Bootstrapping algorithm; Xu Lin-hong [17], based on primary school textbooks, screenplays, literary periodicals and fairy tales, annotated a large amount of Chinese corpus, and formed a "emotional vocabulary ontology library". Although it has been widely used in the field of emotion analysis of Chinese texts, its ontology has some shortcomings in the distribution of corpus and genre Only the timely update of artificial rules and emotional dictionary resources, it can handle new text data well.

So, this paper extended the emotion dictionary and considered the impact of sentence patterns on sentence sentiment in order to achieve more accurate results.

This paper attempted to focus on specific social hot spots and measured the network structure of the public opinion, mined the characteristics of the network structure, and researched the interaction and evolvement mechanism between the main body and the environment in the public opinion propagation. And it introduced the emotion analysis into the evolution of public opinion events, explored the reasons for the sharp fluctuations in emotion throughout the evolutionary life cycle of public opinion, and then provided the decision-making basis for the control of Internet public opinion and the dynamic grasp of public opinion. 


\section{Research on the propagation mechanism of public opinion in social network}

The emergence of social networks provides people with a new information media, but also greatly increases the possibility of the outbreak of public opinion. Public opinion propagation on social networks is influenced by many factors. This paper mainly studied the three aspects of the subject elements of public opinion propagation in social networks, propagation networks and emotional tendencies.

\subsection{The subject elements of public opinion propagation in social network}

Netizens group is the main body of public opinion in the Internet. They have also provided most of the impetus, and they are the main generating force and affected objects of network public opinion. Representative of netizens are mainly divided into the following four groups: grassroots, net-a-porter, network opinion leaders and web hyper.

Grassroots: The grassroots is the most active basic crowd of netizens in china. When grassroots gathered together due to a topic or event that causes a resonance or mood fluctuations, the group leaders assert, repeat, and infect constantly to act on the individual in the way of implication and cross-infected, and they will turn to a common direction, and will immediately turn this concept or belief into action tendencies, so that emotions are greatly vented.

Net-a-porter: Net-a-porter is the netizen who is engaged in forwarding and spreading network information. The existence of the net-a-porter makes network voices amplified infinitely, and the information gets fully widely spread and exchange [10].

Network opinion leaders: The behaviors of network opinion leaders are very active and they have excellent ability of expression. For the majority of netizens, they have a strong influence and tractive effort, and the network has decided to focus on the focus and direction of opinion to a certain extent.

Web hyper: The web hyper is a network planner who popularizes enterprise products, customer brands, events and individuals with the help of the network medium. This purely commercial purpose makes the Internet more complex, and they render it through the secondary reports of the traditional media, so that more netizens who are unknown truth are generally involved, and the topic or the event is very easy to be hype, and even become the network killers that hit businesses and slander the individuals [23].

\subsection{Individual contact process of propagation}

Because of the initiative and interaction of the individual participation, network propagation has special advantages in the aspects of information acquisition and propagation. Individuals in the interpersonal communication are not always passive acceptance of information, and it is not like the real life of the traction of the mass media "agenda setting", and they also play multiple roles, such as publishers and communicators. Because of the strong concern, in fact, some individuals have developed into similar network Medias that have the extraordinary influence. The gap between the network propagation media and the network space is very weak, and they together constitute the diverse individuals in the network space. The interactive propagation among diverse individuals has become the main way to spread the information of network public opinion. In fact, the macro level of diffusion process of network public opinion information is the diverse individuals contact process through the micro level. Specifically, in the emerging social media that represented by social network and micro-blog, emergency network public opinion information always released firstly by a few individuals in the network, and then they were 
forwarded or shared by other individuals in order to communicate one relatively stable group of receiving information. Through a virtual social network, "infected group" scale of network public opinion has been expanded, and then they have the social power that cannot be ignored [1].

\subsection{The design of analytic framework}

To analyze the evolution of public opinion of the social network, it needs to analyze the characteristics of the whole network structure and the characteristics of the key nodes from the two levels. Considering the whole development evolutionary characteristics of public opinion events, we need the evolutionary analysis of time series that is aimed at network event scale, density, average degree, clustering coefficient and so on [2], [4], and we focused on the evolutionary process of public opinion of rapid growth stage and the mature stage and calculated the network structure and the network properties of the important nodes in order to explore the evolution rules of public opinion of the whole network. To understand the emotional state held by users in the process of event information, the user's emotions in the propagation network need to be judged. Based on the above research, this paper first crawled and texted the specific events studied, and divided the text into clauses. In the second step, to segment the text, we needed to create a dictionary of special-purpose emotion words that belongs to the event, based on the existing definition of thesaurus, and marked the sentiment classification of the emotion words, polarity value and its intensity. The third step, combined with the sentence, expression and emotional words of this paper gave the emotional tendency of the text and emotional polarity values.

\section{Construction and analysis of public opinion propagation net- work in social network}

The development of public opinion events is always carried out in the dimension of time. After the formation, proliferation, explosion and termination of public opinion, the social network of public opinion presents the communication of user emotion, attitude, intention and view in turn. On the basis of the research on the overall development of public opinion event of the "Shandong illegal vaccine", in this paper, the social network analysis method based on time series was used to segment the daily relational data in the process of public opinion social network and form the public opinion social network of daily data. Then we analyzed the overall network structure, the evolution of network characteristics and the evolution of key nodes in these different stages.

\subsection{Data source collection and overall structure analysis}

On March 18, 2016, an article entitled "Billions of yuan of vaccine without refrigeration flows into 18 provinces" caused widespread comments and forwarding from users. Micro-blog users expressed their opinions, including celebrities, media, government and other users. The Shandong illegal vaccine case caused public opinion to continue to pay attention. In this incident, what was the attitude of different types of users to events? What was the direction of public opinion? What role did the different users play? Based on this, a study was conducted on the "illegal vaccine". In this paper, the "illegal vaccine" as the keywords for micro-blog search, we collected data from March 18, 2016 to April 4, 2016, and used web crawler software for micro-blog data crawl, and obtained the data through the preparation of crawling rules, a total of 10035 data collection.

Data were cleaned and pre-processed by Excel. Duplicate data, garbled data, incomplete data, and data without records were deleted. After cleaning, 8,222 data were obtained, and we 


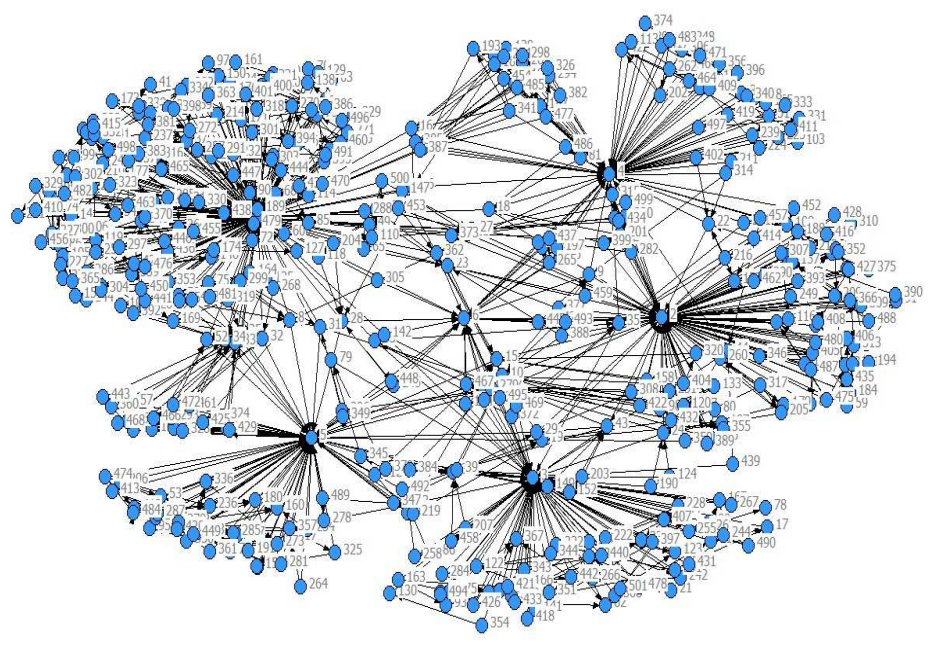

Figure 1: The forwarding network visualization

teased out the micro-blog's forwarding relationship through Sina micro-blog's forwarding rules: if A forwards $\mathrm{B}$, the value of $\mathrm{A}<->\mathrm{B}$ is 1 ; if the two are not forwarded, the value is 0 ; and the main diagonal value of the matrix is defined as 1 . We constructed the public opinion network matrix of "Illegal Vaccine in Shandong". Based on the constructed matrix, visualization software [20] was used to process the forwarding network of the event, and the visualization result was shown in Figure 1.

\subsection{Whole characteristics evolution analysis}

To analyze the evolution of the overall characteristics of the selected cases, the index of public opinion network of daily data of the event needed to be analyzed, according to the trend and regularity of daily public opinion network, and the law of the overall network topological index with event changes was explored.

(1) The evolution of network density

The network density is used to describe the close degree of node interaction. The greater the density of the whole network is, the more frequent the interaction between nodes is. The blue curve in fig. 2 showed the close degree of interaction of each user daily posting and replies in the public opinion event, and the green curve indicated the change of delta value of each variable in two days. It can be found from the curve trend: from March 19 to the end of March, network density had remained flat changing trend. But in April, this topic again appeared fluctuations.

(2) The evolution of network average degree

The blue curve in Figure 3 showed daily users' network average degree of the public opinion event, and the green curve indicated the change of delta value of each variable in two days. From the point of curve trend, the development trend of the network average degree was fluctuating. From March 19 to the end of March, the public opinion event was gradually come to an end from the outbreak of public opinion, the value of network average degree appeared larger fluctuation, especially in the period of hot topic.

The average degree of the whole network became larger and each node had more surrounding nodes for forwarding the interaction, and the communication was more extensive. Namely between March 22-24, the network average degree was higher, it indicated that during this period, 


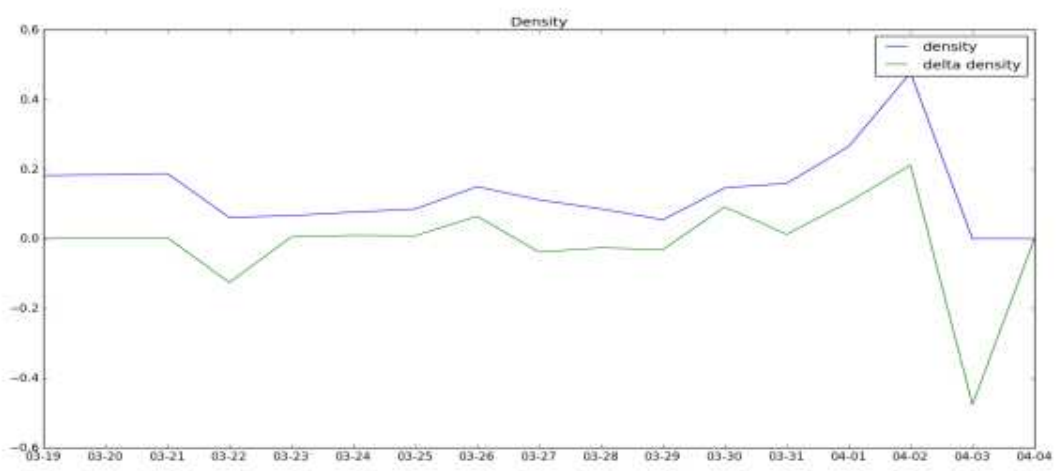

Figure 2: The diagram of network density evolution

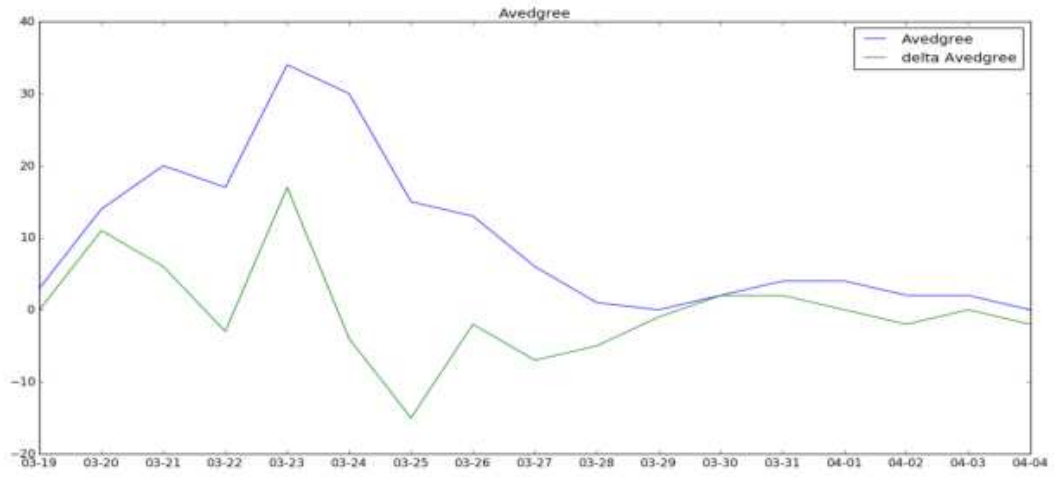

Figure 3: The diagram of the evolution of network average degree

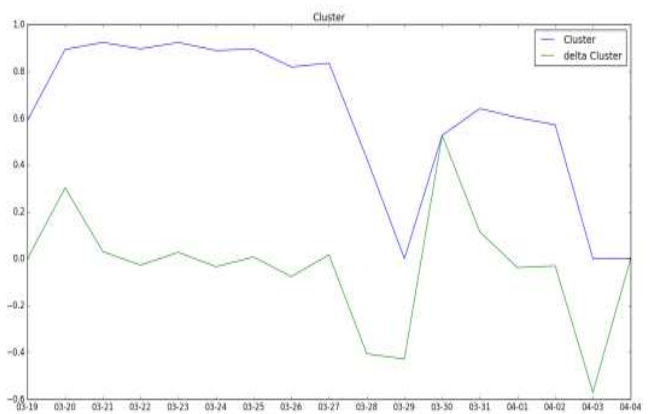

Figure 4: The diagram of the evolution of network clustering coefficient 
many users on the network were repeatedly posting and replying, it may be for a topic or a post and caused a lot of participants to discuss, we can judge, there may be potential opinion leaders.

(3) The evolution of network clustering coefficient

If a node $j$ in the network has $n j$ neighbor nodes, then there may be a maximum of $n j(n j-$ 1) $/ 2$ edges between the $n j$ nodes. We define the ratio of actual number of edges $E(j)$ between nodes $n j$ and the possible number of edges $n j(n j-1) / 2$ as the clustering coefficient of nodes $C C(j)$, that is:

$$
C C(j)=2 E(j) /[n j(n j-1)]
$$

The clustering coefficient $C C$ of the whole network is the average of the clustering coefficients of all nodes $j$ :

$$
C C=\frac{\sum_{j=1}^{N} C C(j)}{N}
$$

Network clustering coefficient describes the degree of network collectivization [6], that is, it is used to identify whether there is a central tendency in the network. Whole network clustering coefficient is bigger, the network of centralized trend is higher, and the users of public opinion tend to the interaction with small groups [7]. The blue curve in fig. 4 showed the daily change of clustering coefficient of the network public opinion, and the green curve indicated the change of delta value of each variable in two days. From the point of curve trend, after the outbreak of public opinion from March 19, it was the first time to achieve the highest value of network clustering coefficient on March 20, and then it entered into a state of smooth fluctuation and maintained between $[0.6,1]$. After March 27, the development of public opinion was into a smooth state, the number of people involved in public opinion and the interaction frequency dropped. In the small batch of node communication, the network clustering coefficient was the same as the network density, which will show a large fluctuation phenomenon.

\subsection{Key nodes characteristics evolution analysis}

In the research of the key nodes in the social public opinion network, scholars often used the static structure characteristics of social network, such as the degree centrality and the structural holes, to analyze the differences in grades and advantages between the key nodes and other nodes in the network. Therefore, based on the public opinion event of "Shandong illegal vaccine",combined with the life cycle theory, we analyzed the characteristics of key nodes in different stages, so as to study the characteristics evolution of network public opinion.

According to the event of "Shandong illegal vaccine", we focused on the characteristics of the key nodes in the rapid growth stage and the mature stage. Statistics showed that the number of people involved existed obvious periodic: after the outbreak of public opinion from March 19, it reached the climax of the public opinion firstly on March 22, and then the growth rate of public opinion had been reduced. It reached the biggest outbreak of the whole public opinion event on March 24, and then it began to subside slowly. Based on this, we set the stage of rapid growth from March 19th to March 22nd, the mature stage, from March 23rd to March 24th.

\section{Degree centrality}

It can be seen from the diagram of the propagation network, the propagation of public opinion presented divergent trend around the individual points, in the propagation network of public opinion, and it showed that there was a strong interaction space around some topics or some 
users. Through measuring degree centrality of this nodes, it can be seen that "The concept of tide", "Friendly 88", "Swineherd is officer","bing-lv" and other users' centrality degrees are bigger, and their participation was relatively high, and it showed some characteristics of opinion leaders. In the two stages of the topic development, different nodes played an important role in the network. In other words, the node was only active before a certain stage, which was not coherent. Therefore, the evolution of a single node cannot be analyzed. Therefore, this section focused on the study of the network structure changes in different stages and the performance of nodes in this stage.

\section{Structural holes}

Structural hole refers to a non-repeating relationship between two nodes, which is equivalent to a buffer in a network. The value of the contribution to the network of the two related people who exist structural holes between nodes can be accumulated. By using UCINET software, we analyzed structural holes in public opinion propagation network of "Shandong illegal vaccine".

It can be seen from the table 1, the public opinion propagation network of "Shandong illegal vaccine" existeda large number of structural holes. We sorted out the effective scale, and "Golden Orchard fruit", "qfy180" and "black feeling" ranked the top three. The value of effective scale reflected the position of nodes in the propagation network, and the bigger the value is, the more core the position becomes. The global constrain of these three nodes was relatively small, they were less than 0.2 , and it also reflected that the three nodes were not easy to be controlled by other nodes and easier to access and spread public opinion. As can be seen from the results of the structural hole in the mature stage, there were still a large number of structural holes in the network of public opinion of "Shandong illegal vaccine". We sorted out the effective scale, and "Happy stock886644", "The Lord of Peach Blossom Island-6" and "Dawn is now tomorrow" ranked the top three. By comparing with the rapid growth stage, it reflected the similar characteristics of the network in different stages of the topic.

Table 1: The structural holes of rapid growth stage

\begin{tabular}{|l|l|l|l|}
\hline Node & Degree & Constrain & Density \\
\hline Golden Orchard fruit & 25.000 & 0.178 & 0.850 \\
\hline qfy180 & 25.000 & 0.178 & 0.850 \\
\hline black feeling & 37.000 & 0.117 & 0.857 \\
\hline Open the treasure to eat & 31.000 & 0.131 & 0.677 \\
\hline Friendly 88 & 36.000 & 0.122 & 0.943 \\
\hline I don't know at all & 22.000 & 0.184 & 1.390 \\
\hline haohuanlea & 11.000 & 0.350 & 0.764 \\
\hline pour out words & 37.000 & 0.117 & 0.857 \\
\hline
\end{tabular}

The analysis showed that in the evolution of network density and network clustering coefficient, the events of rapid growth and maturity showed a relatively steady state, and the trend of its delta curve was basically the same as the original curve. By comparing the network attributes of rapid growth stage and mature stage, the density of networks in mature stage was larger, which indicated that in the mature stage, the overall participation and interaction of network users were more frequent, the propagation distance was shorter and the communication ability was the strongest. In the two stages of topic development, different nodes played an important role in the network. Nodes were more active before a certain stage. Such activities are not coherent. Therefore, it was harder to dismiss the opinion leaders as the whole process of the incident, it needed to identify potential opinion leaders at a finer level of granularity and 
Table 2: The Structural holes of mature stage

\begin{tabular}{|l|l|l|l|}
\hline Node & Degree & Constrain Density \\
\hline Happy stock 886644 & 111.000 & 0.039 & 1.175 \\
\hline The Lord of Peach Blossom Island_6 & 21.000 & 0.217 & 0.543 \\
\hline Dawn is now tomorrow & 102.000 & 0.042 & 1.351 \\
\hline Love the dog & 100.000 & 0.043 & 1.404 \\
\hline $\begin{array}{l}\text { Beijing United Hospital of China and } \\
\text { America }\end{array}$ & 28.000 & 0.158 & 0.860 \\
\hline put quality before quantity & 40.000 & 0.111 & 0.801 \\
\hline Love Wolf & 11.000 & 0.358 & 0.655 \\
\hline ren398540882 & 107.000 & 0.040 & 1.256 \\
\hline
\end{tabular}

conducted early warning of incident developments and public opinion regulation.

\section{The incident text emotional calculation}

As the most popular social network platform, micro-blog users express their opinions and feelings through words and expressions. These messages obviously include emotional information. These sections will statistics emotions and intensity in the whole text from three aspects: emoticons, emotion words and sentence patterns. We analyzed the data of "Shandong illegal vaccine" from March 18, 2016 to March 28, 2016.

\subsection{Emotional dictionary construction}

However, due to the colloquy of micro-blog, a large number of emoticons and related terms for specific events, we constructed an event-specific emotion dictionary. On the basis of comprehensive comparison of existing emotion dictionary, this paper referred to Dalian polytechnic Chinese emotional vocabulary ontology database, which divides the words into two categories: positive emotion ("happy", "good") and negative emotion ("angry", "sad", "evil", "fear", "shock"). Use the word segmentation software to segment the collected text, according to the word frequency from high to low, and then we manually added words that did not come from Dalian Polytechnic dictionary, but also gave emotional classification and emotional strength to these words. The words included not only adjectives, nouns, but also modal word. Part of the expansion of the emotional dictionary is shown.

Table 3: Expansion of emotional dictionary (part)

\begin{tabular}{|l|l|l|l|}
\hline Words & $\begin{array}{l}\text { Emotional classi- } \\
\text { fication }\end{array}$ & $\begin{array}{l}\text { Emotional } \\
\text { strength }\end{array}$ & $\begin{array}{l}\text { Emotional } \\
\text { tendency }\end{array}$ \\
\hline Bully & evil & 5 & negative \\
\hline Affording general satisfaction & good & 9 & positive \\
\hline $\begin{array}{l}\text { Bend the law for the benefit of } \\
\text { relatives or friends }\end{array}$ & evil & 7 & negative \\
\hline Beyond description & angry & 7 & negative \\
\hline Get out & angry & 5 & negative \\
\hline Barbarian & evil & 5 & negative \\
\hline
\end{tabular}

In order to reduce the influence ofthe collocation of positive emotion words or negative emo- 
tion words and negative words, in general, the emotional tendency of positive emotion and negative word tends to be negative emotion, and the emotional tendency of negative emotion and negative word tends to be weak emotion or not Emotions [11]. Therefore, we first determined whether there are negative words adjacent to or similar to the emotional words, in this paper, negative emotions that are negative words modified by negative words, positive emotion words modified by negative words are regarded as negative emotions, negative emotion words modified by negative words are considered as neutral emotions. The negative words include "no", "not", "none", "never", and so on. Emoticons are also commonly used to express emotions in micro-blog. This paper still classified emoticons appearing in texts based on the seven emotions categories in Dalian Polysemous Emotional Dictionary, and the corresponding relationship between emotion classification and expressions is shown in the Table 4.

Table 4: The corresponding table of emotions and emotional categories (part)

\begin{tabular}{|l|l|l|l|}
\hline Emoticonal text & $\begin{array}{l}\text { Emotional classi- } \\
\text { fication }\end{array}$ & Polarity value & $\begin{array}{l}\text { Emotional } \\
\text { tendency }\end{array}$ \\
\hline$[$ Too happy $]$ & happy & 7 & 1 \\
\hline$[$ Haha $]$ & happy & 9 & 1 \\
\hline$[$ Humph $]$ & angry & 3 & 2 \\
\hline$[$ Angry $]$ & angry & 7 & 2 \\
\hline$[$ Retched $]$ & sad & 3 & 2 \\
\hline$[$ Disappointed $]$ & sad & 7 & 2 \\
\hline$[$ Surprised $]$ & shock & 7 & $\mathbf{2}$ \\
\hline SSmiles $]$ & happy & 5 & 1 \\
\hline
\end{tabular}

\subsection{Micro-blog emotional polarity calculation}

The polarity value of each microblog is determined by the emotion word, the sentence pattern and the emoticons in the microblog text. Specific model algorithm steps are as follows:

(1) For each word in the established emotional dictionary, calculate its TF-IDF value with all the text as the total corpus, and mark it as then the emotional polarity of the ith word in this event is: $P W i=F i * P W i$;

(2) The emotional polarity value of each sentence is determined by the emotion words, emoticons and sentence pattern The process of calculating the emotional polarity of a sentence is to calculate the sum of the emotional polarity values of all the emotional words and emoticons in the sentence firstly, and then adding the sentence rules to determine the polarity of the entire sentence. Let $P(S i)$ be the emotional polarity of the sentence before considering the sentence pattern, that is: $P(S i)=\sum S E+\sum P(W)$, where $\sum S E$ is the sum of the emotional intensities of all the emoticons in the sentence, and $\sum P(W)$ is the sum of all the emotion words in the sentence.

(3) Let $P^{\prime}(S i)$ be the sentence emotion value after considering the sentence pattern characteristics, and Table 5 is the sentence emotional polarity weighted rule of six sentence patterns.

(4) The emotional polarity value of a single micro-blog $S i j$ is the sum of the emotional polarity values of each sentence, that is, $P(S i j)=\sum_{k=i}^{j} P^{\prime}(S k)$ 
Table 5: Special sentence calculation instructions

\begin{tabular}{|l|l|}
\hline Sentence pattern & $P^{\prime}(\mathrm{Si})$ \\
\hline Rhetorical questions & $P^{\prime}(\mathrm{Si}) *(-0.6)+(-0.05)$ \\
\hline Interrogative sentence & $P^{\prime}(\mathrm{Si}) *(-0.2)+(-0.05)$ \\
\hline Exclamation sentence & $P^{\prime}(\mathrm{Si}) *(1.5)$ \\
\hline Assumptions sentence & $P^{\prime}(\mathrm{Si}) *(-0.2)$ \\
\hline Declarative sentence & $P(S i)$ \\
\hline Ironically & $-P(S i)$ \\
\hline
\end{tabular}

\subsection{Statistical results}

Emotional calculation was carried out on the collected micro-blog texts of this event, and the emotion word, the sentence pattern and the emoticons were taken into account in the calculation. Emotional values of the event micro-blog texts were calculated through a calculation rule of a single micro-blog text. Statistics on the intensity of micro-blog texts for a single micro-blog text as a Unit were conducted.

Table 6: Positive and negative polarity text statistics

\begin{tabular}{|l|l|}
\hline Negative texts number & Positive texts number \\
\hline $6434(85 \%)$ & $1113(14.7 \%)$ \\
\hline
\end{tabular}

As can be seen from Table 6, negative micro-blog texts occupied most of share, about $0.3 \%$ of the texts did not have emotional values, and $85 \%$ of the texts were negative texts. Negative texts were very unfavorable to the development of the event and the subject of the event, so it was especially important to pay attention to the negative micro-blog in the event. This paper drew the negative polarity intensity figure according to the time (Figure 5). Combined with the release of time-critical information of vaccine events, the dynamic process of emotion trend can be initially determined. The highest value of first-pass negative emotional polarity was reported on March 18 after the outbreak of illegal vaccine, culminating on the $19^{t h}$. The second round of the highest negative emotions appeared at 23rd-24th, which was due to the spread of emotions caused by the widespread spread of "The sorrow of vaccine".

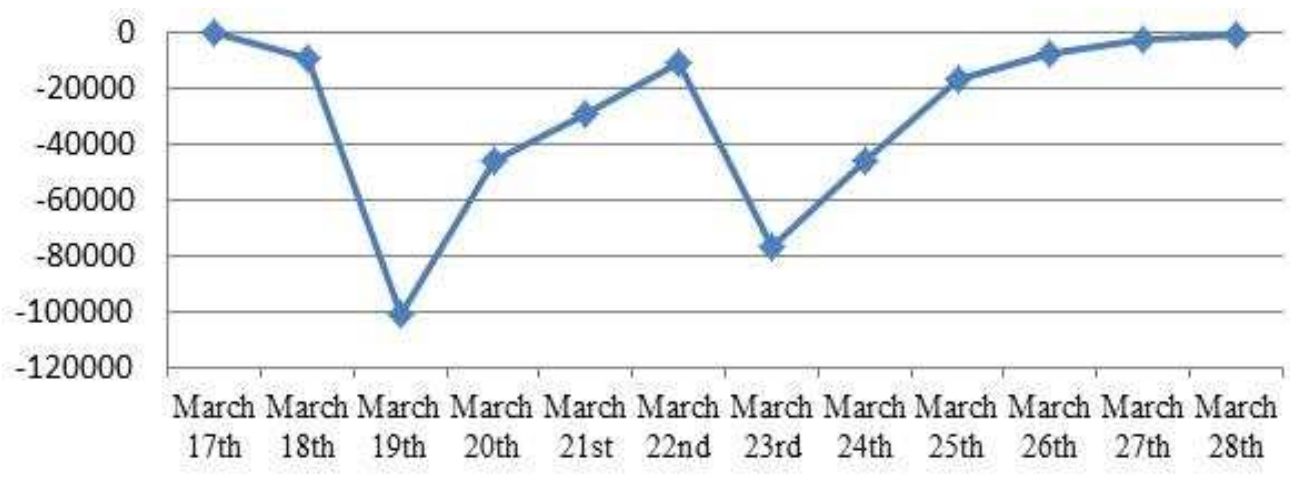

Figure 5: Emotional polarity of Shandong illegal vaccine

The public had a strong emotional response as the incident had many risk characteristics such as affecting children and influencing the population. State Food and Drug Administration, the State Council, the National Health and Family Planning Commission quickly intervened, 
and issued and responded several times, and communicated with the public. From the abovementioned incident, the public opinion and emotion tendency of internet public opinion showed that the public and the media paid close attention to the incident and responded to the progress of the incident handling. The key nodes of incident handling coincided with the peaks of the network public opinion. It was suggested that government departments plaid an important role in the handling of incidents and the release of key information. Timely release of authoritative and transparent objective information was conducive to dredge network public opinion, and it can reduce the negative influence brought by internet public opinion. In this incident, the government sector will definitely not make a clear statement of its impatience and will effectively cut the responsibility so that public emotions will be released and it will be easy to establish and restore trust.

\section{Conclusion}

Based on the theory of network public opinion evolution, this paper took the example analysis of microblog public opinion events, and used the ways of the data crawler, segmentation technology, the construction of emotional dictionary and annotation patterns to get the emotional tendency and emotional polarity of events Then, we explored the causes of the peaks and emotional evolutions that had occurred throughout the evolutionary life cycle of public opinion. The analysis of vaccine events showed that risk perception of the public and society in the media environment tended to deviate easily, and it was difficult to form a self-correcting mechanism. To standardize the processing of media information and to monitor and guide public sentiment is the key to risk communication in crisis And it is a need to speed up the formulation and construction of a system of rules governing the expression of public opinion. The investigation of government and relevant departments' intervened investigation and its result has played an important role in the development of public opinion. The timely announcement of the government and the factual release of the truth in real time were conducive to resolving the contradictions and quelling the public opinion. In the information era, the government and relevant departments should not expect to reduce the spread by covering up with negative news And openly and fairly dealing with them according to law, which is the basic requirement of a society ruled by law.

Although this paper is a single case study of recent typical risk events, the illegal vaccine event is a microcosm of the outbreak of public opinion in recent years. Lack of rigorous media information and the extensive emotional evolution in the network have a direct impact on risk perception. The changes of network structure and emotional polarity exhibited during the amplification of the risk of illegal vaccines have empirical value in coping with other similar social focus events.

\section{Acknowledgment}

Work described in this paper was funded by the National Natural Science Foundation of China under Grant No. 71671093 and the National Natural Social Foundation of China under Grant No. 16ZDA054. The authors would like to thank other researchers at Nanjing University of Posts and Telecommunications.

\section{Bibliography}

[1] Chen Y.J. (2010); An Effective Way to Explore the Internet, Computer and Information Science (ICIS), 529-534, 2010. 
[2] Cho, Y.; Hwang, J.; Lee, D. (2012); Identification of effective opinion leaders in the diffusion of technological innovation: a social network approach, Technological Forecasting and SocialChange, 79(1), 97-106, 2012.

[3] Han, S.C.; Liu, Y.; Chen, H.L.; Zhang, Z.J. (2016); Influence Model of User Behavior Characteristics on Information Dissemination, International Journal of Computers Communications \& Control, 11(2), 209-223, 2016.

[4] Hong, X.J.; Jiang, N.; Xia, J.J. (2014); Study on Micro-blog Rumor Based on Social Network Analysis - A Case Study on the Micro-blog about Food Security, Intelligence Journal, 8, 161-167, 2014.

[5] Lee, C. H. (2012); Mining Spatio-temporal Information on Micro-blogging Streams Using a Density-based Online Clustering Method, Expert Systems with Applications, 39(10), 96239641, 2012.

[6] Nair, H.S.; Manchanda, P.; Bhatia, T. (2010); Asymmetric Social Interactions in Physician Prescription Behavior: The Role of Opinion Leader, Journal of Marketing Research, 47, 883-895, 2010.

[7] Paltoglou, G. (2016), Sentiment-based Event Detection in Twitter, Journal of the Association for Information, Science and Technology, 67(7), 1576-1587, 2016.

[8] Riloff, E.; Patwardhan, S.; Wiebe, J. (2006); Feature Subsumption for Opinion Analysis. Conference on Empirical Methods in Natural Language Processing, 440-448, 2006.

[9] Su, X.-P.; Song, Y.-R. (2015); Leveraging neighborhood "structural holes" toidentifying key spreaders in social networks, Acta Phys. Sin, 2, 5-15, 2015.

[10] Sznajd, W.K. (2006); Opinion Evolution in Closed Community, Physics C, 11, 1157-1165, 2000 .

[11] Wang, L.; Zhao, Y.; Liang, S.H. (2013), Micro-blog Social NetworkAnalysis Based on Network Group Behavior, Advanced Materials Research, 79(12), 435-438, 2013.

[12] Wen, F.; He, Z.; Dai, Z.; Yang, X. (2014); Characteristics of Investors' Risk Preference for Stock Markets, Economic Computation and Economic Cybernetics Studies and Research, 48(3), 235-254, 2014.

[13] Wen, F.; Gong, X.; Cai, S. (2016); Forecasting the volatility of crude oil futures using HAR-type models with structural breaks, Energy Economics, 59, 400-413, 2016.

[14] Wen, F.; Xiao, J.; Huang, C.; Xia, X. (2018); Interaction between oil and US dollar exchange rate: nonlinear causality, time-varying influence and structural breaks in volatility. Applied Economics, 50(3), 319-334, 2018.

[15] Wiebe, J.; Riloff, E. (2012); Finding Mutual Benefit between Subjectivity Analysis and Information Extraction, IEEE Transactions on Affective Computing, 2(4), 175-191, 2012.

[16] Xiong, F.,; Liu, Y.; Zhang, Z.J. (2012); An information diffusion model based on retweeting mechanism for online social media, Physics Letters A, 6, 2103-2108, 2012.

[17] Xu, L.; Lin, H.; Zhao, J. (2008); Construction and Analysis of Emotional Corpus, Journal of Chinese Information Processing, 1, 116-122, 2008. 
[18] Zhang, D. (2017): High-speed Train Control System Big Data Analysis Based on Fuzzy RDF Model and Uncertain Reasoning, International Journal of Computers Communications \& Control, 12(4), 577-591, 2017.

[19] Zhang, H.; Small, M.; Fu, X. (2011); Staged Progression Model for Epidemic Spread on Homogeneous and Heterogeneous Networks, Journal of Systems Science and Complexity, 24, 619-630, 2011.

[20] Zhang, D.; Sui, J.; Gong, Y. (2017); Large scale software test data generation based on collective constraint and weighted combination method, Tehniki vjesnik, 24(4), 1041-1049, 2017.

[21] Zhao, J.L.; Cheng, J.H. (2015); Analysis of Micro-blog Public Opinion Diffusion Based on SNA: AN Empirical Study on April 20 Ya'an Earthquake in Sichuan, Management Review, 1, 148-157, 2015.

[22] Zhao, H.Q. (2015); Analysis of Complex Network Public Opinion Communication, Journal of Qinghai University (Natural Science), 4, 29-37, 2015.

[23] Zhou, M.; Liu, X.; Pan, B. (2017); Effect of Tourism Building Investments on Tourist Revenues in China: A Spatial Panel Econometric Analysis, Emerging Markets Finance and Trade, 53(9), 1973-1987, 2017. 